\title{
Caracterização Físico-Química da Superfície de Filmes de Poli(Tereftalato de Etileno)
}

\author{
Georgia V. V. V. Pinto, César A. M. Abreu, Augusto Knoechelmann e Yêda M. B. Almeida
}

Resumo: Dentre as diversas técnicas de caracterização superficial de filmes poliméricos, foram utilizadas nos filmes de PET do presente estudo a refratometria, rugosidade, análises de coeficiente de atrito e ângulo de contato. As amostras de filmes de PET analisadas têm $12 \mu \mathrm{m}$ de espessura e compreendem três tipos de estuturas superficiais: filme de homopolímero PET, filme PET com tratamento corona e filme de poli(tereftalato de etileno-co-ciclohexanodimetanol). O filme com tratamento corona apresentou o maior valor de tensão superficial em função da maior parcela dos grupos polares. Resultados de índice de refração indicam que a superfície do filme com copoliéster é menos cristalina que as superfícies dos demais filmes avaliados. A estruturas amorfa e elevado nível de tensão superficial de filmes tratados deverão favorecer a um aumento dos níveis de adesão a tintas e revestimentos.

Palavras-chave: Poli(tereftalato de etileno), caracterização, morfologia, rugosidade, filme.

\section{Introdução}

A caracterização de superfícies poliméricas vem se tornando muito importante nos últimos anos, em função do crescente aumento de aplicações e interações entre os materiais poliméricos e os produtos industrializados em geral (Ishitani, 1984). Tratamentos superficiais são frequentemente empregados para atender, por exemplo, ao mercado de embalagens flexíveis (Pengilly, 1982-1983).

O estudo de alguns fenômenos envolvidos, como a forma em que um líquido se espalha sobre uma superfície, a rugosidade de um material, os grupamentos e as ligações superficiais e a adesão entre filmes poliméricos, ou entre um filme e um revestimento, têm sido bastante explorados (Mizerovskii,1992; Galembeck,1991). Associadas a cada um desses fenômenos existem propriedades mensuráveis, capazes de fornecer interpretações qualitativas e quantitativas dos mesmos e que estão diretamente relacionadas com a performance industrial do material.

A fim de avaliar estas propriedades, técnicas de análise de superfície vêm sendo desenvolvidas e estão se tornando ferramentas de grande valia na compreensão destes fenômenos. No presente estudo foram analisados três filmes de poli[tereftalato de etileno] com $12 \mu \mathrm{m}$ de espessura, com diferentes tratamentos superficiais. A grande importância deste trabalho está em se utilizar técnicas precisas e mutuamente complementares que apresentem informações detalhadas a respeito destas superfícies uma vez que a caracterização química e física não têm sido feita de uma forma 
profunda e abrangente, havendo poucos trabalhos com resultados globais.

\section{Materiais e Métodos}

Os filmes estudados foram fornecidos pela Terphane Ltda (Cabo-PE). O filme A não apresenta tratamento superficial; o filme B foi tratado por descarga corona, com energia normalizada de $20 \mathrm{~J} / \mathrm{cm}^{2}$ e o filme C apresenta uma camada da ordem de 0,4 $\mathrm{mm}$ de espessura de poli[tereftalato de etileno-cotereftalato de ciclohexanodimetanol], com 84,6\% do monômero tereftálico e 15,4 \% do comonômero.

As análises de índice de refração foram realizadas num refratômetro digital tipo Abbe, da marca Mark / Reichert Jung, da Leica. As análises foram feitas nas três direções convencionalmente adotadas ( $\mathrm{x}, \mathrm{y}$ e $\mathrm{z}$ ). Os valores de rugosidade bidimensional foram obtidos num rugosímetro Hommelwerke, modelo T2000, sendo o apalpador de diamante (com raio de $5 \mu \mathrm{m}$ ). Os resultados foram obtidos em termos dos parâmetros $R_{a}, R_{z} e$ $R_{t}$ e representam a média de 100 amostras.

As análises de coeficiente de atrito foram realizadas segundo a ASTM D 1894, procedimento B, no aparelho da Daventest.O método da gota séssil discutido por Garbassi et al. (1994) foi escolhido para analisar os filmes PET num goniômetro Ramé-Hart NRL modelo 100-00 equipado com acessório 100-15 para filmes plásticos. Os líquidos de referência utilizados nestas análises foram o a-bromonaftaleno (P.A) como líquido apolar e água destilada e deionizada (Milli Q) como líquido polar. O ângulo de inclinação da base para medidas dos ângulos de avanço e de retrocesso foi de $50^{\circ}$; estas análises foram realizadas 6 meses após a produção dos mesmos, sendo que durante este período os filmes foram mantidos a $25^{\circ} \mathrm{C}$. A metodologia utilizada foi descrita por Pinto (1998).

\section{Resultados e Discussão}

Os índices de refração fornecem informações importantes com relação à orientação e morfologia das cadeias poliméricas superficiais. A Tabela 1 apresenta os resultados de cristalinidade (X), birrefringência $(\Delta n)$, polarisabilidade $(P)$, percentual de cadeias no sentido longitudinal
Tabela 1. Parâmetros de orientação e cristalinidade dos Filmes A, B e C.

\begin{tabular}{cccc}
\hline & Filme A & Filme B & Filme C \\
\hline Cristalinidade $(\%)$ & 47,19 & 45,93 & - \\
Birrefringência & 0,0186 & 0,0198 & 0,00 \\
$\Delta \mathrm{n} 12$ & 0,1710 & 0,1714 & 0,00 \\
$\Delta \mathrm{n} 13$ & 0,1524 & 0,1516 & 0,00 \\
$\Delta \mathrm{n} 23$ & 0,0823 & 0,0823 & 0,0700 \\
Polarisabilidade $(\mathrm{P})$ & 0,3346 & 0,3346 & 0,3145 \\
$\mathrm{P}_{\text {2ax/MD }}$ & 0,1617 & 0,1615 & 0,0002 \\
$\Delta \mathrm{P}$ & & &
\end{tabular}

$\left(\mathrm{P}_{\text {2ax/MD }}\right)$ e orientação planar das $\operatorname{cadeias}(\Delta \mathrm{P})$ dos filmes $\mathrm{A}, \mathrm{B}$ e $\mathrm{C}$, que são parâmetros calculados à partir destes índices.

A cristalinidade foi calculada através da Eq.1, sendo que os coeficientes foram obtidos segundo Le Bourvellec \& Beautemps, especialmente para filmes PET:

$$
X(\%)=(18,2 \mathrm{~N}-28,7) 100
$$

A birrefringência é um parâmetro que mede o grau de orientação molecular, e é calculada de forma simplificada através da diferença entre cada dois dos três índices de refração segundo as três direções $\mathrm{x}, \mathrm{y}$ e $\mathrm{z}$ convencionadas:

$$
\Delta \mathrm{n}_{\mathrm{xy}}=\left(\mathrm{n}_{\mathrm{x}}-\mathrm{n}_{\mathrm{y}}\right) ; \Delta \mathrm{n}_{\mathrm{xz}}=\left(\mathrm{n}_{\mathrm{x}}-\mathrm{n}_{\mathrm{z}}\right) ; \Delta \mathrm{n}_{\mathrm{yz}}=\left(\mathrm{n}_{\mathrm{y}}-\mathrm{n}_{\mathrm{z}}\right)
$$

As contribuições das polarisabilidades das unidades moleculares das amostras são obtidas através do índice de refração médio, ou seja:

$$
\begin{aligned}
& \mathrm{N}^{2}-1 / \mathrm{N}^{2}+2=4 / 3 \pi \mathrm{P} \\
& \mathrm{P}=\mathrm{m} \beta
\end{aligned}
$$

com $\beta$ a polarisabilidade de uma unidade molecular, $\mathrm{m}$ o número de moléculas por unidade de volume e $\mathrm{N}$ o índice de refração médio.

Já o percentual de cadeias orientadas no sentido longitudinal à produção do filme de PET $\left(\mathrm{P}_{2 \mathrm{ax} / \mathrm{MD}}\right)$ e a orientação planar dos anéis aromáticos também são calculados respectivamente através das Eq. (5) e (6):

$$
\begin{aligned}
& \mathrm{P}_{2 \mathrm{ax} / \mathrm{MD}}=\left\{\mathrm{n}_{1}-\left[\left(\mathrm{n}_{2}-\mathrm{n}_{3}\right) / 2\right]\right\} 0,21 \\
& \Delta \mathrm{P}=\left[\left(\mathrm{n}_{1}+\mathrm{n}_{2}\right) / 2\right]-\mathrm{n}_{3}
\end{aligned}
$$

Em relação à morfologia, verificou-se pelos valores de cristalinidade que o filme $\mathrm{C}$ é menos cristalino que os filmes A e B, o que pode ser justifica- 
do pela presença do grupo CHDM no copoliéster, que ao gerar impedimento estérico, ocasiona uma composição amorfa da camada externa do filme C. Verificou-se ainda que o filme B é menos cristalino que o filme $\mathrm{A}$, o que pode ser esclarecido ao se assumir que esta amorfização ocasionada pelo tratamento corona é semelhante à amorfização gerada por um excimer laser, discutida por Dunn \& Ouderkirk (1990); segundo eles, mudanças nos espectrogramas obtidos por espectroscopia no infravermelho de reflexão-absorção (IRRAS) indicam que a radiação converte uma grande fração cristalina do filme numa fração amorfa.

Através dos valores de birrefringência $(\Delta n)$, pode-se notar que o filme $C$ não apresenta orientação preferencial, já que as birrefringências encontradas são nulas e que os filmes A e B são um pouco mais orientados na direção transversal que na longitudinal e bem mais orientados nas direções transversal e longitudinal que na normal. Os valores de birrefringência são importantes na determinação das condições de produção de filmes, uma vez que a birrefringência aumenta com a taxa de estiragem do filme, quando processados a temperaturas superiores a $120^{\circ} \mathrm{C}$.

As polarisabilidades dos filmes A e B são iguais e superiores às do filme $C$, mostrando que os filmes A e B apresentam maior capacidade de polarizar a luz. Já os filmes A e B apresentam os mesmos percentuais de cadeias no sentido longitudinal de produção do filme $\left(\mathrm{P}_{2 \mathrm{ax} / \mathrm{MD}}\right)$, sendo superiores ao do filme $\mathrm{C}$, mostrando o menor nível de orientação longitudinal do filme $\mathrm{C}$.

As orientações planares $(\Delta \mathrm{p})$ dos filmes A e B são bem próximas entre si e pelo fato de serem positivas, caracterizam uma anisotropia dos filmes A e $\mathrm{B}$, indicando que as moléculas estão relativamente orientadas e que esta orientação é fixa, não estando livres para girarem em torno de sua posição de equilíbrio. Já no filme $\mathrm{C}$, a inexistência da planaridade indica que as moléculas estão orientadas ao acaso e o meio comporta-se macroscopicamente como uma substância isotrópica (Cardoso, 1994).

Para comparar as taxas de cristalinidade superficiais dos filmes obtidos via índice de refração com a cristalinidade $(\mathrm{X})$ do filme como um todo, abrangendo interior e superfície, foi utilizada uma coluna de gradiente de densidade (Sharma \& Misra, 1987); os valores dados a seguir representam a média de $\mathrm{k}$ amostras de cada filme, sendo s a incerteza.
Tabela 2. Valores de densidade e cristalinidade dos Filmes A, B e C obtidos via coluna de gradiente de densidade.

\begin{tabular}{cccc}
\hline & Filme A & Filme B & Filme C \\
\hline $\begin{array}{c}\text { Densidade } \\
(\mathrm{g} / \mathrm{cm} 3)\end{array}$ & 1,3999 & 1,3975 & 1,3999 \\
$\mathrm{X}=2, \mathrm{~s}=0)$ & $\mathrm{k}=3, \mathrm{~s}=0,0004)$ & $(\mathrm{k}=3, \mathrm{~s}=0)$ \\
$\mathrm{X}(\%)$ & 54,1 & 52,1 & 54,1 \\
\hline
\end{tabular}

Observando a Tab. 2, pode-se dizer que a fina camada $(0,4 \mu \mathrm{m})$ de copolímero do Filme $C$ não foi detectada pelo método de densidade, uma vez que foram iguais às cristalinidades encontradas para os três filmes; isto pode ter ocorrido pois em termos percentuais seu caráter amorfo foi pouco representativo em relação à estrutura total do filme que é semi-cristalina.

Comparando a Tabela 1 com a Tabela 2, podese dizer que as taxas de cristalinidade das superfícies dos filmes são diferentes das envolvendo seus interiores. O filme $\mathrm{C}$ é amorfo em sua superfície enquanto seu interior é semi-cristalino; os filmes A e B são menos cristalinos na superfície do que em seu interior, sendo no entanto estas diferenças da ordem de $15 \%$. Esta menor a cristalinidade superficial proporciona melhores interações entre os filmes e as tintas ou revestimentos, facilitando a penetração e inchamento do revestimento na matriz polimérica. No entanto, deve-se esclarecer que o percentual de cristalinidade de um filme polimérico não pode ser medido de forma absoluta porque o polímero é constituído de regiões amorfas e cristalinas cujas fronteiras não são bem definidas. Deste modo, para calcular a cristalinidade, assumiu-se através de uma aproximação que o polímero é constituído de regiões amorfas e cristalinas bem distintas. Deduções quantitativas entre o índice de refração e a orientação, para melhor caracterizar um filme biestirado, necessitam de análises complementares feitas por outras técnicas, como Espalhamento Raman e técnicas de espectroscopia no infravermelho.

Os valores de coeficientes de atrito (COF) estático e dinâmico dos filmes A, B e C encontramse na Tabela 3.

Os maiores valores dos coeficientes de atrito estático e dinâmico encontrados para o filme B

Tabela 3. Coeficientes de atrito estático e dinâmico dos filmes A, B e C.

\begin{tabular}{cccc}
\hline & Filme A & Filme B & Filme C \\
\hline COF estático & $0,525 \pm 0,022$ & $0,561 \pm 0,034$ & $0,517 \pm 0,023$ \\
COF dinâmico & $0,386 \pm 0,018$ & $0,404 \pm 0,021$ & $0,390 \pm 0,019$ \\
\hline
\end{tabular}


Tabela 4. Valores parciais e totais das tensões superficiais dos Filmes A, B e C.

\begin{tabular}{cccc}
\hline Filmes & $\begin{array}{c}\boldsymbol{\gamma}_{\mathbf{s}}^{\mathbf{p}} \\
(\mathbf{m N} / \mathbf{m})\end{array}$ & $\begin{array}{c}\boldsymbol{\gamma}_{\mathbf{s}}^{\mathrm{d}} \\
(\mathbf{m N} / \mathbf{m})\end{array}$ & $\begin{array}{c}\boldsymbol{\gamma}_{\mathbf{s}}^{\mathrm{T}} \\
(\mathbf{m N} / \mathbf{m})\end{array}$ \\
\hline Filme A & $5,97 \pm 0,15$ & $43,87 \pm 0,06$ & $49,84 \pm 0,06$ \\
Filme B & $9,07 \pm 0,74$ & $43,90 \pm 0,06$ & $52,97 \pm 0,71$ \\
Filme C & $6,27 \pm 0,21$ & $43,83 \pm 0,06$ & $50,10 \pm 0,12$ \\
\hline
\end{tabular}

podem estar associados a maior parcela dos grupos polares presentes na superfície deste filme, que promovem interações do tipo dipolo entre as superfícies deslizantes.

Os ângulos de contato foram utilizados para calcular as componentes dispersivas $\left(\gamma_{\mathrm{s}}{ }^{\mathrm{d}}\right)$ e polares $\left(\gamma_{\mathrm{s}}{ }^{\mathrm{p}}\right)$ da tensões superficiais dos filmes A, B e C (Good, 1970):

$$
\begin{aligned}
& \gamma_{\mathrm{L} 1}\left(1+\cos \theta_{1}\right)=2\left(\gamma_{\mathrm{s}}^{\mathrm{d}} \gamma_{\mathrm{L} 1}{ }^{\mathrm{d}}\right)^{1 / 2}+2\left(\gamma_{\mathrm{s}}{ }^{\mathrm{p}} \gamma_{\mathrm{L} 1}{ }^{\mathrm{p}}\right)^{1 / 2} \\
& \gamma_{\mathrm{L} 2}\left(1+\cos \theta_{2}\right)=2\left(\gamma_{\mathrm{s}}{ }^{\mathrm{d}} \gamma_{\mathrm{L} 2}\right)^{\mathrm{d}}{ }^{1 / 2}+2\left(\gamma_{\mathrm{s}}{ }^{\mathrm{p}} \gamma_{\mathrm{L} 2}{ }^{\mathrm{p}}\right)^{1 / 2}
\end{aligned}
$$

Somando estes componentes dispersivos aos polares, encontra-se a tensão superficial total $\left(\gamma_{s}^{T}\right)$ de cada filme, cujos valores estão listados na Tabela 4.

Dentre os filmes analisados, o filme B apresenta os maiores valores de tensão superficial parcial (das componentes dispersivas e polares) e total. Segundo Leclercq et al. (1977), o maior valor da tensão superficial total do filme B é devido ao tratamento corona introduzir espécies hidrofílicas que alteram a componente polar. As tensões superficiais das componentes dispersivas $\left(\gamma_{\mathrm{s}}^{\mathrm{d}}\right)$ dos filmes A, B e C estão de acordo com Mizerovskii (1992), os quais devem se situar entre 41,0 e $53,6 \mathrm{mN} / \mathrm{m}$, sendo o valor teórico igual a $44 \mathrm{mN} / \mathrm{m}$.

Os ângulos de avanço e retrocesso com água destilada e deionizada foram avaliados (Tabela 5) para verificar a heterogeneidade do filme B e para efeito comparativo, foi analisado o filme A; podese concluir que os filmes analisados são diferentes entre si uma vez que o tratamento corona provoca uma queda dos ângulos de avanço e retrocesso e ao mesmo tempo um aumento na diferença entre estes ângulos, ocasionando uma maior histerese.

Tabela 5. Ângulos de avanço e de retrocesso dos filmes A e B.

\begin{tabular}{lccc}
\hline Filmes & $\begin{array}{c}\text { Ângulo de avanço } \\
\left({ }^{\circ}\right) \text { água }\end{array}$ & $\begin{array}{c}\text { Ângulo de retrocesso } \\
\left({ }^{\circ}\right) \text { água }\end{array}$ & Histerese \\
\hline Filme A & $69,35 \pm 0,88$ & $67,70 \pm 0,59$ & 1,65 \\
Filme B & $61,67 \pm 2,72$ & $59,68 \pm 2,72$ & 1,99 \\
\hline
\end{tabular}

Através da Tabela 5, observa-se que os ângulos de avanço e retrocesso do filme B diferem sensivelmente entre si e também do ângulo de contato em equilíbrio medido a $0^{\circ}$, sendo estes valores inferiores aos do filme sem tratamento. Estes resultados assemelham-se aos de Strobel (1992), cujos ângulos de avanço de filme de PET não-tratado foi $70^{\circ}$ enquanto o do filme de PET tratado por corona foi $64^{\circ}$, sendo bastante próximos aos encontrados respectivamente nos filmes A e B do presente estudo. Estes resultados sugerem uma heterogeneidade da superfície do filme B.

Deve-se considerar ainda que a grande diminuição do ângulo de retrocesso do filme $\mathrm{B}$ favorece à molhabilidade e portanto à adesão da superfície, justificando o emprego do corona na superfície de filmes poliméricos destinados à impressão.

Dentre os diversos parâmetros de rugosidade descritos na literatura, destacam-se $R_{a}, R_{z}$ e $R_{t}$ (Catier,1978). A rugosidade média $\left(R_{a}\right)$ é a média aritmética das distâncias entre a linha média (eixo x) e cada ponto do perfil de rugosidade da superfície, sendo calculada segundo a equação abaixo:

$$
\mathrm{R}_{\mathrm{a}}=1 / \operatorname{lm} \int_{0} \operatorname{lm}|\mathrm{y}(\mathrm{x})| \mathrm{dx}
$$

$O$ valor $R_{a}$ de filmes poliméricos pode ser visto como um indicador do crescimento da microestrutura superficial (Wefers \& Schollmeyer, 1993).

A rugosidade total $\left(\mathrm{R}_{\mathrm{t}}\right)$ compreende a distância entre o ponto mais alto dos picos e o mais baixo dos vales e o terceiro parâmetro de rugosidade denominado por $\mathrm{R}_{\mathrm{z}}$, é obtido dividindo-se o perfil de comprimento total $\left(l_{t}\right)$ estudado em cinco partes iguais de comprimentos $\left(1_{0}\right)$, tomando-se a maior distância em cada intervalo $(Z)$ e fazendo-se a média aritmética, e pode ser calculado segundo a equação (10):

$$
\mathrm{R}_{\mathrm{z}}=1 / 5\left(\mathrm{Z}_{1}+\mathrm{Z}_{2}+\mathrm{Z}_{3}+\mathrm{Z}_{4}+\mathrm{Z}_{5}\right)
$$

Os valores obtidos encontram-se na Tabela 6 .

Os dados de rugosidade fornecidos pela Tabela 6 podem ser considerados iguais para os três filmes em estudo. Este método não foi capaz de detectar diferenças de rugosidade entre os filmes A, B e C, sugerindo a realização de AFM e SEM para melhor visualização da topografia superficial. 
Tabela 6. Rugosidade bidimensional dos filmes A, B e C.

\begin{tabular}{cccc}
\hline Rugosidade $(\boldsymbol{\mu m})$ & Filme A & Filme B & Filme C \\
\hline $\mathrm{Ra}$ & $0,0316 \pm 0,0037$ & $0,0315 \pm 0,0044$ & $0,0330 \pm 0,0050$ \\
$\mathrm{Rz}$ & $0,3388 \pm 0,0259$ & $0,3384 \pm 0,0269$ & $0,3459 \pm 0,0288$ \\
$\mathrm{Rt}$ & $0,6102 \pm 0,0565$ & $0,6128 \pm 0,0595$ & $0,6193 \pm 0,0568$ \\
\hline
\end{tabular}

\section{Conclusão}

Através dos resultados obtidos, pode-se concluir que tanto o filme B com tratamento corona quanto o filme $\mathrm{C}$ com copoliéster devem proporcionar melhores condições de processamento nas indústrias de transformação do que o filme A sem tratamento superficial. Esta melhoria de performance dos filmes tratados em relação ao filme $\mathrm{A}$ sem tratamento se deve a certas propriedades superficiais diferenciadas destes filmes, como ao aumento da tensão superficial do filme $\mathrm{B}$ e à amorfização superficial do filme $\mathrm{C}$, tornando-os capazes de fornecer melhores níveis de adesão a tintas e revestimentos.

\section{Agradecimento}

À CAPES - Fundação Coordenação de Aperfeiçoamento de Pessoal de Nível Superior pelo suporte financeiro em forma de bolsa concedida e a Terphane Ltda. pela confecção das amostras e análises realizadas.

\section{Referências Bibliográficas}

1. Cardoso, V.L - Estudo da estabilidade temporal e do processo de relaxação molecular de filmes poliméricos com propriedades ópticas não lineares, Campinas-SP. Tese de Doutorado, UNICAMP (1994).

2. Catier, E.- La mesure de rugosité : comparateurs, palpeurs, ordinateurs. EAI, 252, (1978).

3. Dunn, D. S. \& Ouderkirk, A. J.- Chemical and Physical Properties of Laser-modified Polymers. Macromolecules, 23, 770-774, (1990).

4. Galembeck, F - Superfícies de Polietileno, suas características e sua adesão. Polímeros: Ciência e Tecnologia, Nov./Dez., (1991).
5. Garbassi, F.; Morra, M.;Occhiello, E.- Polymer Surfaces: from physics to technology. London: John Wiley \& Sons, (1994).

6. Ishitani, A.- FT-IR as a tool for the characterisation of industrial materials. Polymer Preprints: 25, 2, 186187, (1984).

7. Le Bourvellec, G. \& Beautemps, J.- Stretching of PET films under constant load II.Structural Analysis. Journal of Applied Polymer Science: 39, (1990).

8. Leclercq, B., Sotton, M., Baszkin, A; TERMINASSIAN-SARAGA, L.- Surface modification of corona treated (polyethylene terephtalate) film: adsorption and wettability studies. Polymer: 18, 675-680, (1977).

9. Mizerovskii, L. N - Calculation of the contact wetting angle of polymers. International Polymer Science and Technology: 19, 4, 38-40, (1992).

10. Pengilly, B. W. \& Hill, J. W.- Thermoplastic Polyester, Modern Plastic Enciclopedia, 1982-83.

11. Pinto, G. V. V. V. - Efeito do tratamento corona e do 1,4-ciclohexanodimetanol nas características superficiais do filme de poli(tereftalato de etileno) Recife - PE. Tese de Mestrado, UFPE (1998).

12. Sharma, S. K \& Misra, A - The effect of stretching conditions on properties of amorphous polyethylene terephtalate film. Journal of Applied Polymer Science, 34, (1987).

13. Strobel, M.; Lyons, C. S.; Strobel, J. M ; Kapaun, R. S.- Analysis of air-corona treated polypropylene and poly(ethylene terephthalate) films by contact angle measurements and X-ray photoelectron spectroscopy. J. Adhesion Sci. Technol, 6, 4, 429443, (1992).

14. Wefers, L. \& Schollmeyer, E.- Surface characterization of laser-treated poly(ethylene terephtalate) by optical profilometry and scanning tunneling microscopy, Journal of Polymer Science: Part B: Polymer Physics, 31, 23-27, John Wiley \& Sons, (1993). 